\title{
Cloud-based Instruction Model for Electrical Engineering Courses: A Rapid Response to Enable Fully Online Course Delivery
}

\section{Dr. Praveen Meduri, California State University, Sacramento}

Dr. Praveen Meduri is an Assistant Professor of Electrical and Electronic Engineering at Sacramento State University. He is also a Technical Liaison to Cadence Design Systems. He received his PhD from Old Dominion University, VA, M.S. from Southern Illinois University at Carbondale and bachelors from JNT University, India.

His research interests include Embedded Systems, Smart Cities and VLSI Design and has multiple peer reviewed publications in these fields.

\section{Mr. Lawrence David Landis, Intel Programmable Solutions Group}

Senior Manager University Academic Outreach, Intel Programmable Solutions Group

Lawrence has 35 years' experience in a wide variety of functions in the electronics industry including marketing, sales and project management for numerous ASIC and FPGA products. Larry teaches part time digital electronics and ASIC design at Santa Clara University and UC Berkeley.

\section{Prof. Perry L. Heedley, California State University, Sacramento}

PERRY L. HEEDLEY earned his Ph.D. and M.S. degrees from Auburn University and his B.E.E. from the Georgia Institute of Technology. He has over 20 years of industrial experience designing analog and mixed-signal integrated circuits for companies including Intel, Sacramento (formerly Level One Communications), Crystal Semiconductor, and Harris Semiconductor. In 2003 he joined the Department of Electrical and Electronic Engineering at California State University, Sacramento, while continuing to consult with industry on a part-time basis. In 2005 he co-founded the Mixed-Signal Design Laboratory (MSDL) at Sacramento State with Professor Thomas W Matthews. His interests include high-speed data converters, low-jitter clock generation and distribution, high-speed serial data communications, switchedcapacitor circuits for analog signal-processing, low-voltage analog design in deep sub-micron CMOS processes, and mixed-signal circuits for biomedical applications.

\section{Mr. Tyler Sheaves, Intel Corporation}

Tyler Sheaves is a Ph.D. student at University of California, Davis and a graduate technical intern in the academic outreach division of Intel's Programmable Solutions Group. In his position at Intel, he develops virtual FPGA-based learning applications and curriculum. In academia, his primary areas of research are hardware security, intellectual property protection, and digital integrated circuit design and verification. 


\title{
Cloud Based Instruction Model for Electrical Engineering Courses - A Rapid Response To Enable Fully-Online Course Delivery
}

\begin{abstract}
In this paper, we present four techniques that have been successfully adopted for delivering labbased electrical and computer engineering courses, in a fully-online format. This experiment is a rapid response to the exigent circumstances necessitating a sudden transition to online instruction format.
\end{abstract}

Firstly, we present the use of public cloud based servers for hosting custom IC design software. This software (for example, Cadence Virtuoso suite) is essential to impart important learning outcomes and to ensure curricular currency in electrical and computer engineering programs.

This approach has relevance that extends beyond the upper division class (Digital VLSI Design) in which it is implemented. This could be a model for enabling fully online course delivery in engineering; particularly in courses that have significant compute-intensive simulation needs. We also discuss the specifications and compute resource optimizations of cloud server instances in relation to the class size. The long-term viability of this cloud-based model for online instruction is considered based on the user feedback, added value and cost metrics.

Secondly, we present a cloud-based computational platform (called DevCloud from Intel), for use in heterogeneous computing courses. Knowledge of heterogeneous computing systems has been identified by IEEE Computer Society and ACM as a core learning outcome in computer engineering curricula [1]. The paradigm of heterogeneous computing entails a judicious combination of CPUs and co-processor accelerators like FPGAs or GPUs. This significantly accelerates arithmetic intensive workloads (like Artificial Intelligence projects), by leveraging the power of data-level and task-level parallelism.

Thirdly, we also present a couple of tried and tested approaches for delivering hardware based electrical and computer engineering courses in online format. These FPGA lab related approaches have been successfully implemented in courses at different universities worldwide.

Even as different universities plan a phased return to in-person instruction, we feel confident in asserting that these approaches will have an enduring positive role in creating a robust educational system that can disseminate hands-on electrical engineering skills, without regard for geographical limitations.

\section{Introduction}

As universities grapple with the sudden need to embrace fully online instruction modality, the agility of the transition process is constrained by the ability to move labs and other hands-on learning content into remote delivery format. Particularly, engineering courses which have significant lab needs are less agile and less prepared. There is an increased urgency to find both quick, make-shift solutions and also long-lasting ones. The lessons learned and practices adopted 
during these exigent times can have long-lasting positive impacts on how online education is delivered.

The prevailing covid-19 situation has exposed the fault lines and also spurred tremendous innovation in our education system. While the negative impacts of this malady are enormous (1.6 billion students effected worldwide, UNO, [2]), with ripple effects and aftershocks likely to continue in the near future, that it has inspired and accelerated all around innovation in the delivery of education is undeniable.

In this paper, we present four techniques that have been successfully adopted specifically for delivering lab-based courses in online format.

These techniques provide options for readily embracing online modality with very little friction. Our observation is that the systems that already had the necessary infrastructure in place before the pandemic are the ones with the least amount of friction during transition.

\section{Contributions and organization of this paper}

The main contributions of this paper are:

(i) In section - II, we present a public cloud-based approach to deliver compute intensive engineering lab courses in a fully online format.

(ii) The cost vs value analysis for public cloud based solutions is also presented. Some implementation particulars are provided, with our Digital VLSI course as a running example.

(iii) In section - III, we feature a cloud based computational platform for heterogeneous computing courses. The computational platform called DevCloud (by Intel) has been adopted my numerous universities. We provide GitHub links to download ready-touse, fully-developed coursework in heterogeneous computing.

(iv) In section - IV, we also present 2 techniques to deliver hardware (FPGA development kit) based engineering labs online.

(v) In effect, these approaches present options that have been successfully tried in delivering both software and hardware-based engineering labs in online format; there by satisfying a sine qua non for a frictionless transition to fully online course delivery.

We begin with a short review of the existing work on cloud-based engineering education solutions.

\section{Brief Literature Review - Cloud Based Engineering Education}

There are already a handful of works published in this still unfolding situation. For example, [3] discusses a futuristic cloud based education model, informed by a local study done on the impact of corona virus. In [4] a strategic three phase response to navigate the uncertain times is presented, along with a vision for best education solutions and a shift towards a more student centered learning paradigm.

There is a preponderance of pre-pandemic literature on approaches to cloud-based engineering education. This is consistent with our observation that the systems that had least friction in 
transferring to online format are the ones that already had the necessary infrastructure in place, for example, [5].

Quadri, Et Al. investigated into the critical success factors for cloud based e-learning approach [6] using a combinatorial approach. They also proposed a framework based on 14 factors for success that are grouped into 4 dimensions. In [7], the main factors for adoption of cloud-based technologies in higher education are identified. The correlation between cloud-based technologies and Bloom's Taxonomy based learning objectives has been investigated. Although their research focuses on a broader scope of higher education, their insights are relevant in engineering discipline. Particularly, the interplay between the observable factors and latent factors that influence the adoption of cloud based technologies is highlighted in insightful manner. However, particulars about lab-based engineering courses are not in the scope of this paper. The benefits and challenges of cloud computing services in education system are presented in [8]. A typical architecture that is representative of cloud-based e-learning systems is depicted in [7].

A systematic study of published research in the area of cloud computing [9] for education has been presented in 2018 in [10]. Their review finds that as of published work till 2016, 'Virtual Laboratories' is one of the topics of interest. Based on a relatively high number of conference papers compared to the number of journal papers, the authors conclude that the research into cloud-based education solutions is in its nascent stages. They also identify that most of the research published on cloud-computing for education has been "conceptual" or "non-empirical", with fewer number of papers providing empirical evidence based on fully implemented solutions.

For courses that have purely simulation/virtual lab components several cloud based approaches have been suggested [11], [12], [13], [14], [15] and most of them are based on running software on virtual machines and/or virtualization on private or public cloud servers.

Augustin, Et Al., demonstrate a virtual lab management system that can handle both simulation based and hardware based course needs in engineering courses [16]. In [17], a dynamic online lab system that can control remotely connected CNC machines, LEDs and servo motors is presented. An approach to provide a seamless integration of actual and remote labs for FPGA based hardware design has been presented in [18]. [19] presents an interesting Lab-as-a-Service solution.

From our research it is our opinion that the solutions available for lab-based courses that have the empirical backing of successful implementation are few. As such, the current paper demonstrates a few techniques that have been successfully implemented and presents low risk, independent solutions for instructors to make quick changes in their courses with minimal disruption to the chain of command.

A good analysis of 4 of the public cloud services vendors is presented in [20]; with particular emphasis on the costs aspects of these options. Even though the information is from 2014, the general "pay-per-use" pricing model of these public vendors is well analyzed in the article. 
Efforts to build an integrated Micro Electronics Cloud Alliance (MECA) based on public, private and hybrid cloud services (as defined by NIST [9]) have been reported in [21]. MECA reports the use of all levels of cloud services including Infrastructure as a Service (IaaS), Platform as a Service (PaaS) and Software as a Service (SaaS) that have been identified in [9]. A comparative discussion on the advantages and disadvantages of both private and major public cloud service providers is presented in [22].

\section{Cadence Virtuoso tools on the Cloud - A case study}

In this section, we present a cloud-based approach that has been tried in our Digital VLSI course. Specifically, we hosted Cadence Virtuoso tools on public cloud-based servers. Our approach is presented in this section. Some details will be provided about (i) outsourcing the installation and maintenance related IT tasks, (ii) operational aspects, (iii) overall user reception of this approach and also about (iv) the cost $v s$ value considerations of this approach.

With minor operational modifications, this approach can be applied as a generic model for many engineering courses that have compute-intensive lab components. It's a modular cloud-based solution that can be rapidly deployed to address specific course needs. We begin with a brief description of the Digital VLSI course, as a running example.

\section{Running Example - Digital VLSI Course}

The Digital VLSI course constitutes an important component in upper division electrical and computer engineering curriculum in VLSI (Very Large Scale Integration) and System-On-Chip (SoC) design. In addition to in-class instruction, this course has significant lab (circuit simulation) component that involves schematic \& layout design and verification steps. Industry standard Cadence Virtuoso tools are extensively used in the lab simulations in the course.

Many similar courses like Analog VLSI, Mixed-Signal VLSI and other SoC related courses share a similar curricular model. In fact, this project-based learning paradigm of in-class instruction, reinforced through team projects is representative of many engineering courses. In [23], an engineering team-project based learning approach to mentoring graduate students through their master's projects in presented.

In the Fall semester of 2020, the Digital VLSI course had an enrollment of 55 students, and it has been taught in a fully online, synchronous format; owing to the suspension of in-person instruction. This course is cross listed between graduate and undergraduate levels. The cadence assignments were divided into 2 projects, each with multiple phases including schematic simulation, layout, DRC and LVS and other verification steps of a custom design workflow for SoCs. Most of the students had reasonable experience using circuit simulation software like multisim, but little to no experience with Cadence Virtuoso tools. Detailed step-by-step instruction videos have been created in lieu of in-person lab instructions. The course has been delivered synchronously with teaching assistants conducting scheduled lab help sessions remotely through zoom sessions. 


\section{Traditional Approach vs Cloud Based Approach - Motivation}

In a traditional scenario of on-campus instruction, these Cadence Virtuoso set of tools are installed on university owned servers and the infrastructure is maintained by the resident IT group of the engineering college/university. Students using these tools would need to login to the servers from on-campus computers and invoke the tools on premises. When required to access the university servers form off-campus via a VPN service, a significant lag is experienced.

In order to mitigate this issue, we deployed cloud servers to run the Cadence Virtuoso tools (as a substitute for on campus servers) for use in a Digital VLSI class. Students can access the servers and invoke the Cadence tools in the cloud, by logging in from their personal computers. Since routing via a VPN network is not necessary in this model, a significant bottleneck is eliminated thereby improving the overall user experience with this compute intensive tool. Additional advantages of this cloud-based approach are discussed later in this section.

\section{IT Technical Support and EDA Tool Administration - Outsourced to CMC Microsystems}

The installation and maintenance of the license server and the required tool chain in the cloud can be handled by resident IT support group of the college or university. Alternatively, Cadence Cloud Passport Partner program [24], supported by Cadence allows for these installation and maintenance services to be outsourced to one of their approved consulting partners.

We chose to retain the services of a Cadence approved cloud partner, for a nominal fee. This consulting firm, CMC Microsystems [25], is a non-profit organization that specializes in providing software services to help reduce barriers to technology adoption. Other Cadence approved cloud consulting partners are listed on [24].

The Cadence Virtuoso tools themselves could be hosted on many of the public cloud vendors like Amazon Web Services (AWS), Microsoft Azure, Google Cloud, etc. In this project, the cadence servers are hosted on AWS.

\section{Advantages of the Cloud Based Approach}

The overall look and feel of the Cadence Virtuoso tool in AWS cloud has been very similar to that installed on university servers. However, there are significant advantages to the AWS approach.

Firstly, the need to route through the university's VPNs is eliminated, as mentioned above. This contributes to a significant improvement in performance and responsiveness of the compute intensive graphical tool, thereby improving the overall user experience.

Secondly, unlike the static university servers, public cloud-based servers have on demand access to the most up-to date processor and other compute resources; without waiting for a lengthy refresh cycle, which is around 5 years, typically. The time overhead of quoting, procuring, and configuring are eliminated leading to a solution that is rapidly deployable.

Thirdly, there is a two-fold advantage to outsourcing the IT Support services: (i) this approach does not burden the in house IT support system that is probably already stretched and (ii) the 
consulting firm can install and configure the most up-to date software tool chain necessary for the course workflow.

Furthermore, this cloud-based approach provides a quick way to enable fully online course delivery by providing server access to students worldwide.

The efficacy of this approach has been successfully demonstrated in a large scale experiments [5] at the DAC (Design Automation Conference) summer school in July 2020.

\section{Operational Details}

The consulting company, CMC worked out an appropriate configuration for the cloud servers based on the expected course enrollment and workflow requirements. Specifically, 2 servers each with 32 GB RAM and 8 vcore general purpose processors have been deployed in the AWS cloud, in time for the beginning of the semester.

The annual Cadence license agreement can be extended at no additional cost - to function in the cloud servers. CMC handled all the necessary operational details like automatically downloading the control file, license management and the necessary toolchain installation with all the dependencies. The requisite Cadence toolchain was installed and tested in a development and test environment before committing to the production environment.

Once the AWS servers are deployed in production environment for class use, student's login using a secure shell SSH X11 client like VNC Viewer. As an optional layer to enhance security, a public/private key based on SHA security scheme can be implemented. However, since the course projects did not involve sensitive IP or other proprietary data, the login was secured using a user id and password. For students using Mac machines, login can be through Mac's native terminal and xquartz programs. Students were responsible to back-up their work before the cloud servers were shut-down at the end of the semester.

One small implementation improvement to the approach could be to use a dynamic load balancing scheme to auto assign users to one of the two compute nodes, instead of statically dividing the users among the two servers. It is worth noting here, as an aside, that when the full load (of 55 students) was presented to just one server as an experiment, the speed and performance degraded, and the system crashed during peak traffic time. The second server was quickly deployed and the class load distributed evenly. With second server, all the students could use the Cadence tools at the same time, without any reduction in performance.

\section{Overall User Experience}

Overall, the experience in AWS cloud has been positive as indicated by qualitative student feedback. Students reported significant improvement in the responsiveness of the tool, even when accessing from a thin client. Feedback has been solicited from users with prior experience in traditional approach and the general consensus has been that AWS server provided a much smoother experience, as shown in Chart-1 and Chart- 2 below. The authors understand that it is not appropriate to generalize based on a data set of around only 60 users and that the lack of 
controlled experiments does not lend statistical backing to the results. However, the user feedback in terms of responsiveness of the tool, speed of execution and overall user experience has been clearly positive.

Student experience - Traditional VPN approach

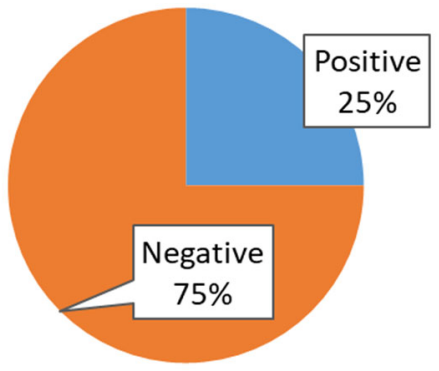

Student experience - Cloud based approach

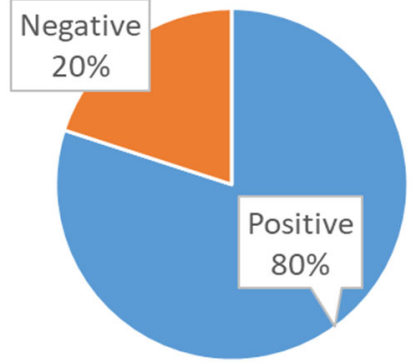

Chart - 1 Overall student experience: Traditional approach vs Cloud-based approach

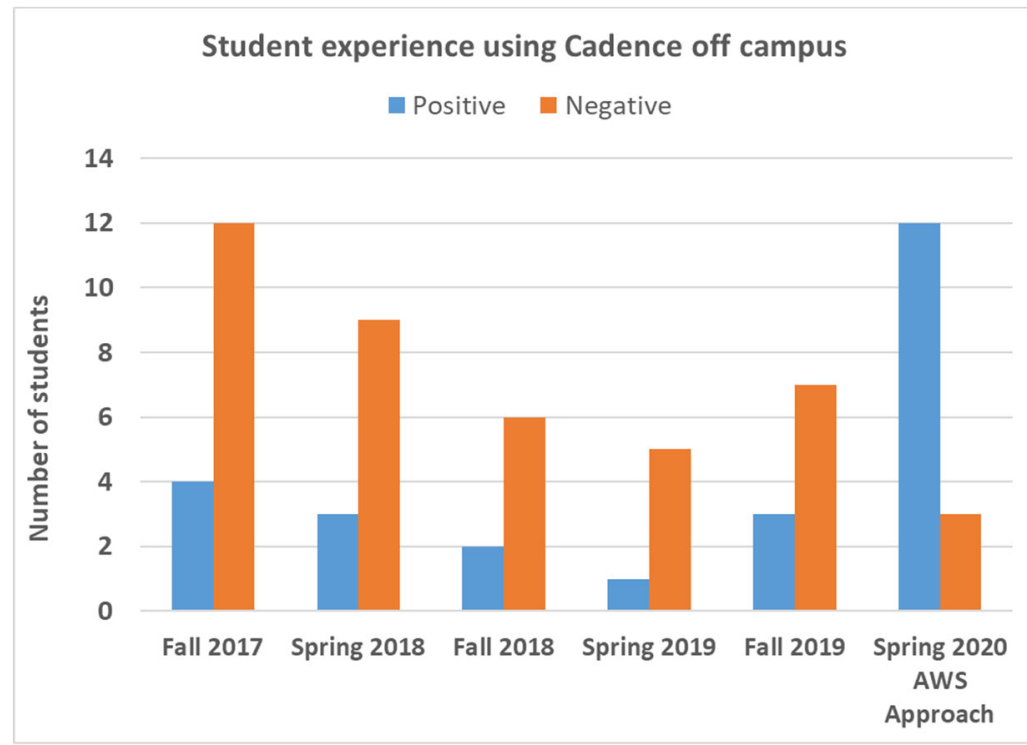

\section{Chart - 2 Student experience comparison over semesters}

This data has been collected from the end of semester student evaluations. Specifically, only those comments pertaining to responsiveness of the tool, speed of execution and overall user experience while accessing Cadence off campus have been curated and categorized as positive or negative. Most of the projects have been very similar across the different semesters and the lab manual has been updated periodically. As an aside, some of the positive reception of the AWS approach could be attributed to the fact that students were given additional resources like stepby-step video to ease the process of accessing the cloud server and also because of increased TA support in the virtual labs. Nonetheless, the cloud based approach provides a much better overall user experience. 


\section{Cost vs Value Considerations}

In this section, we provide some details about the costs involved and the pricing model for AWS cloud-based approach. The costs listed below are in addition to the licensing costs for the Cadence tools themselves.

(i) Consulting company costs: The consulting company, in this case CMC, charges a nominal per-year fee for the IT support services.

(ii) Amazon's Elastic Computing Costs: These are server hosting charges billed to CMC on an hourly use basis by AWS and are passed through to the licensing university without any mark-up. Typical AWS charges for different configurations are shown in the table-1 below. (Source: CMC estimate). CMC exploits multiples optimizations at the organization level to bring the overall costs below the hourly pricing model shown in Amazon's website [26].

\section{Table - 1 Estimated monthly expense for AWS server instance (estimate by CMC)}

\begin{tabular}{|c|c|c|}
\hline vCPU \# of cores & Memory (GB) & Estimated monthly cost/instance \\
\hline 4 & $16 \mathrm{~GB}$ & $\$ 225.00$ \\
\hline 8 & $32 \mathrm{~GB}$ & $\$ 350.00$ \\
\hline 16 & $32 \mathrm{~GB}$ & $\$ 625.00$ \\
\hline 16 & $64 \mathrm{~GB}$ & $\$ 700.00$ \\
\hline
\end{tabular}

As can be calculated from the table- 1 above, the cost per year (12 months) for a typical AWS configuration using 2 instances (each with 8 core vCPUs and 32 GB RAM) is approximately $\$ 8000$. Incidentally, out of this, the first $\$ 2000$ were offset by CMC during the first year of service with them.

For comparison purposes, we also provide some details about the costs in the traditional approach. An estimate of the cost for a physical server for a traditional on premises use is around $\$ 17000$ for a PowerEdge R7425server with 256 GB RAM and 64 vCPU cores (quick estimate provided by the IT Department of the author's university). For institutions that rely on college wide Computing Services/IT departments, the infrastructure cost is typically budgeted into the IT department's expenditure and does not represent a direct cost to the individual courses. Given that the typical lifetime of such a server is 5 years, the annualized costs for a server of equivalent specs (16 vCPU and 128 GB RAM) can be approximated to be between $\$ 500$ and $\$ 600$. This is much lower compared to the 'pay-per-use' AWS estimate of $\$ 8000$. However, the traditional server involves a larger initial investment by the organization.

Thus, if considering only the procurement costs for the on premises server, the AWS cloud approach entails a considerably smaller upfront expenditure. It would seem that the overall return 
on investment in the AWS does not match that from a physical server over the typical life span of the infrastructure. However, taking into account all the operational and hidden costs involved with the physical server, a total cost of ownership analysis shows that cloud based approach could save up to $30 \%$ in overall costs to the university [27].

Furthermore, it is possible to reduce the yearly usage costs (to around $\$ 5000$ for 30 weeks or 2 semesters), by turning off the AWS servers in between the semesters or when the course is not in session. This however, did not present an attractive choice for us, because we have a significant number of students who use the tools outside the regular semester.

In effect, while taking into account only the procurement costs of the server, and considering only those direct costs that are incurred by the course, the traditional on-premises servers are significantly less expensive. Of course, the exact processor architectures and specifications are not matched for these estimates. Hence the forgoing analysis is only meant for understanding the general pricing models and approximate costs. Additional operational costs of the on-premises servers are significant [27], but are not directly billed to the department offering the course. They are not discussed here.

We presented a cloud-based solution for delivering fully online engineering courses that have significant compute-intensive lab needs. The long-term viability of this approach is to be determined after careful consideration of all the costs involved and the advantages of the approach.

\section{Heterogeneous Computing Course - Intel's FPGA DevCloud}

In this section, we feature a cloud-based computational platform called DevCloud, for use in heterogeneous computing courses.

Knowledge of heterogeneous computing systems has been identified by IEEE Computer Society and ACM as a core learning outcome in computer engineering curricula [1]. The paradigm of heterogeneous computing entails judicious combination of CPUs and co-processor accelerators like FPGAs or GPUs. This significantly accelerates arithmetic intensive workloads (like Artificial Intelligence projects), by leveraging the power of data-level and task-level parallelism.

Intel's FPGA DevCloud [28] is an excellent cloud-based platform that provides the compute resources required in heterogeneous computing courses. This free cloud service offered by Intel's Programmable Solutions Group offers Xeon processors and FPGA Platform Acceleration Cards (PAC) to universities teaching heterogeneous computing. The typical workloads supported on DevCloud include RTL (Verilog/ System Verilog/VHDL), and high-level synthesis languages OpenCL and OneAPI ( $\mathrm{dpc}++$ language). This free cloud environment now services thousands of members.

The DevCloud launched in July 2019, and with the onset of Covid-19, the rate of new subscribers tripled from March 2020 to April 2020 and on, coincident with Covid-19 stay at home orders. Members are able to compile workloads and download the workload accelerator image on to FPGA cards. Compilations do not necessarily need to be run on the same nodes that 
have attached PAC cards. Access to development tools can be through interactive GUI, command line, or can even be launched in batch mode.

To increase access and coursework targeted at FPGA DevCloud users, Intel offers grants to professors to generate heterogeneous computing curriculum work. Published work includes RTL acceleration [29], OpenCL [30] and OneAPI/dpc++ [31] and is available as GitHub repositories. Many universities are using their own or sponsored labwork. $\mathrm{PhD}$ level research is also commonplace on the Intel FPGA DevCloud.

Intel chose to host their own cloud service versus utilizing AWS or alternatives after exploring the cost/benefit analysis of such a setup. The use of cloud services provides academia low overhead access to expensive servers and FPGA PAC cards, while offering high productivity for the user community.

\section{Digital Logic Design Coursework: Hands-Free Remote Console and Labsland}

In this section, we present two FPGA remote hardware access approaches successfully used in undergraduate Digital Logic Design courses.

The first approach, called the hands-free remote console, is based on virtualizing hardware (FPGA development development kits from Intel) so that students can remotely interact with the development kit's tactile inputs (such as buttons/switches) and view outputs (such as LEDs and seven segment displays). This scheme enables users to interact with a remotely connected FPGA development kit through a realistic GUI medium shown in Figure 1. The user manuals and example projects for rapid adoption of this scheme are available on GitHub [32].

The remote console is in use at numerous universities and at Intel's training sites utilizing a local connection of Quartus host machine directly to the development kit, or alternatively to a farm of kits connected to a single Linux server host running JTAG connectivity to multiple development kits. Some examples of universities hosting the remote console setup include UMass Lowell, San Francisco State University, and Temple University.

The second approach is a camera-based implementation that captures the state of LEDs through a video camera feed. The tactile input to FPGA is stimulated through a GPIO connection by a Raspberry Pi board that captures mouse clicks from the UI and stimulates input switch activity.

\section{Hands-Free Remote Console - Implementation Details}

In this sub-section, some implementation details of the Hands-Free Remote Console are presented.

Intel's FPGAs offer a dedicated JTAG interface in their devices for programming the device, debug of the FPGA through signal probing, connectivity to embedded processors, and instrumentation of FPGA hardware. The JTAG interface provides a portal to monitor the state of inputs and set the state of outputs. PCs do not have a native JTAG bus connection, so a bridging "Blaster" circuit on the development kit converts the signals from USB protocol to JTAG 
signaling. The JTAG interface is used to monitor signals on the FPGA development kit and remotely recreate a realistic GUI package.
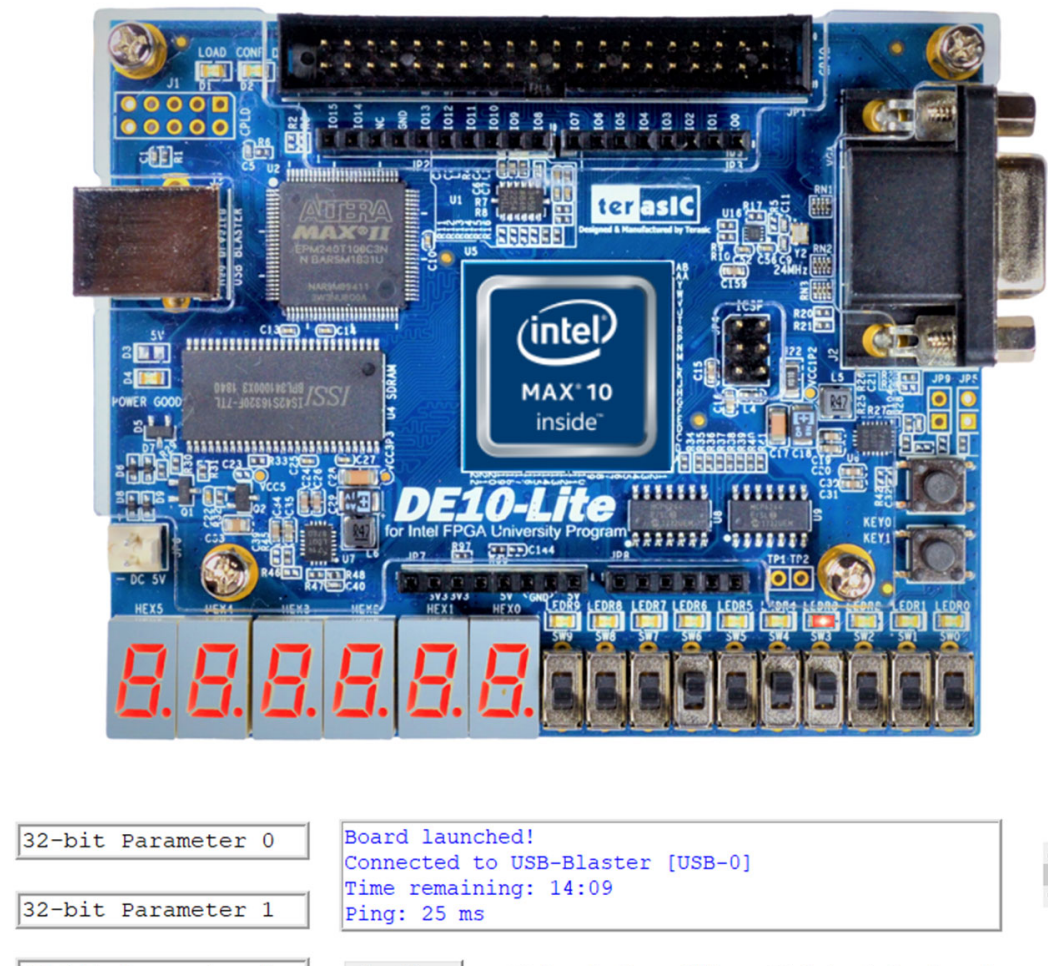

Reset Timer $\nabla$ Input from GUI $\Gamma$ output to Board

Figure 1: DE10-Lite FPGA Development Kit Remote Console - GUI

Intel's FPGA Quartus Prime development tools include an application called Platform Designer that can be used to connect master and slave devices to the on-chip bus. Platform Designer uses the Avalon bus (similar to an AXI bus) to plug in a vast array of Intel supplied master/slave IP functions. Functions required for the remote console monitoring function include a JTAG bus master block and various memory mapped GPIO blocks to stimulate inputs and monitor outputs of the student's design.

The second Quartus Prime application used to create the remote console experience is called System Console. System Console uses TCL commands to read and write the memory space created by Platform Designer.

Launching the remote console application as shown in Figure 2 enables a user to connect locally or network hosted FPGA hardware development kits. The user specifies local or IP address, the name of the programming image file (.sof) and launches the remote FPGA development kit. Clicking on moveable objects such as slide switches and push buttons change their state which in turn serve as FPGA stimulus while the pin_ip block monitors expected behavior response to LEDs and seven segments. 


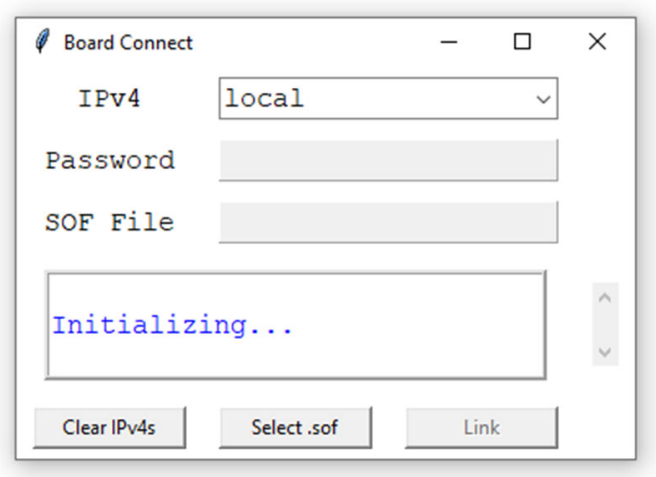

Figure 2: Input local or IPv4 address and associated programming image file

To integrate a user function, the student connects their user function to Platform Designer IP block to monitor inputs and drive outputs.

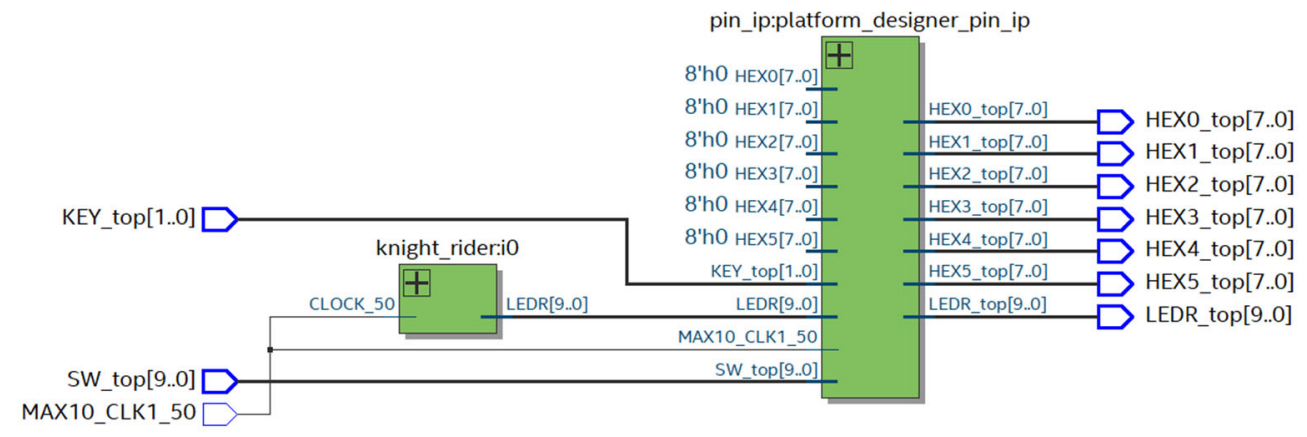

Figure 3: User function connected to pin_ip block which monitors input and drive output activity through memory mapped IO.

The remote console offers a realistic interface to actual FPGA hardware without need for a simulator, presenting unique advantages such as running at full clock speed, and the inclusion of rich debug functions such as the Signal Tap logic analyzer tool.

\section{Camera based remote control and monitoring of FPGA development Kits}

Another innovative means to connect to remotely hosted FPGA development kits for online learning of Digital Logic Design is offered through a company called Labsland [33], [34]. Labsland is a collection of remotely hosted labs on a variety of scientific topics. Their business model is to utilize learning institutions to host the remote labs, and Labsland collects a per student fee to access the remote learning setup. Host sites acquire free access to the remote labs. Examples of hosted labs are Arduino powered robots, FPGA compilation and hardware download, radioactivity measurements and many more. US based host sites for Digital Logic Design coursework using FPGAs include University of Washington and University of Michigan.

Labsland uses a simplified IDE to run Intel FPGA Quartus development tools "under the hood" and remove some of the complexities of FPGA hardware design typically associated with project 
setup, constraints, and design creation. Alternatively, students can create their programming image .sof file using their locally hosted version of Quartus tools and download their programming image to the remotely hosted FPGA development kit.

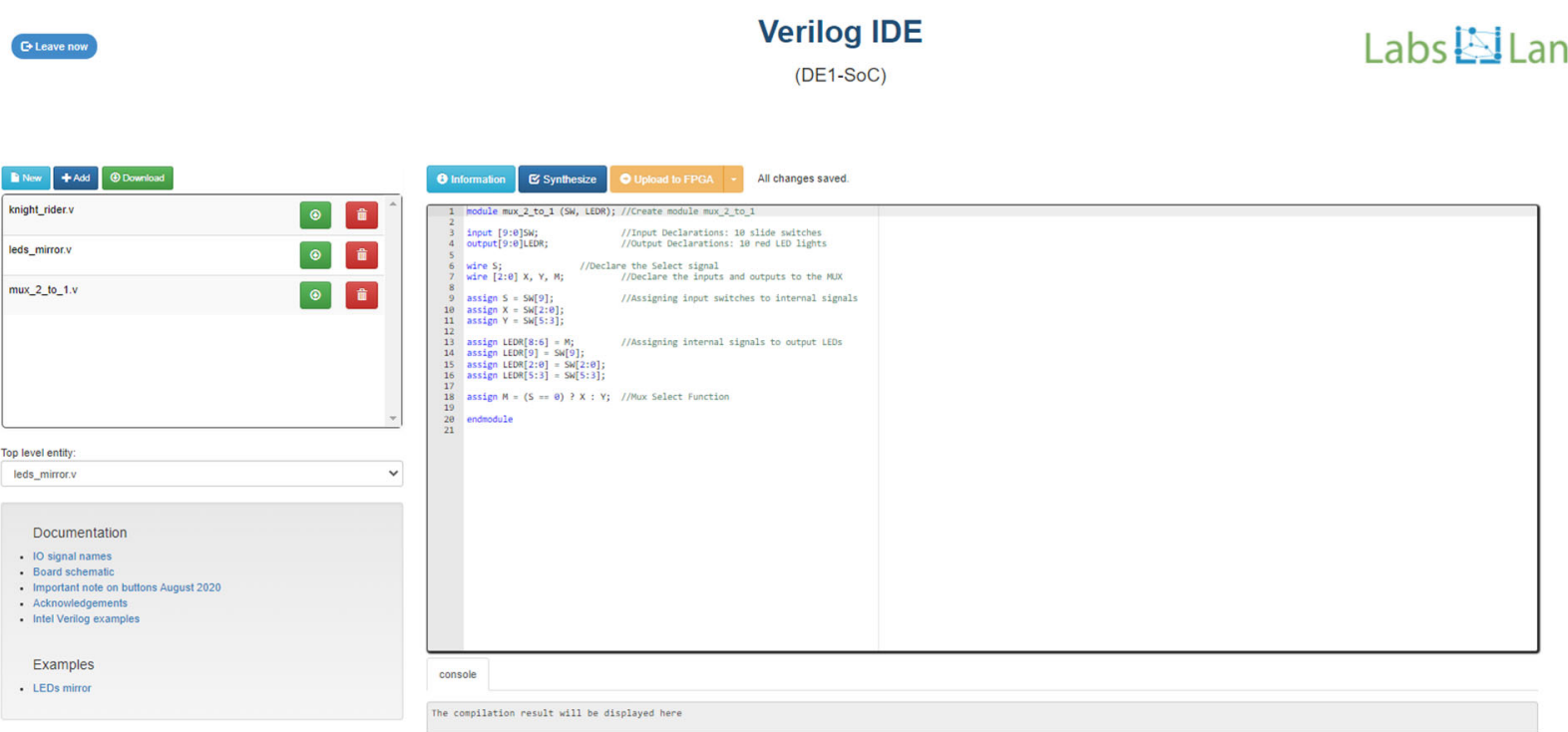

Figure 4: Labsland Verilog FPGA IDE for the Terasic DE1-SoC FPGA development kit
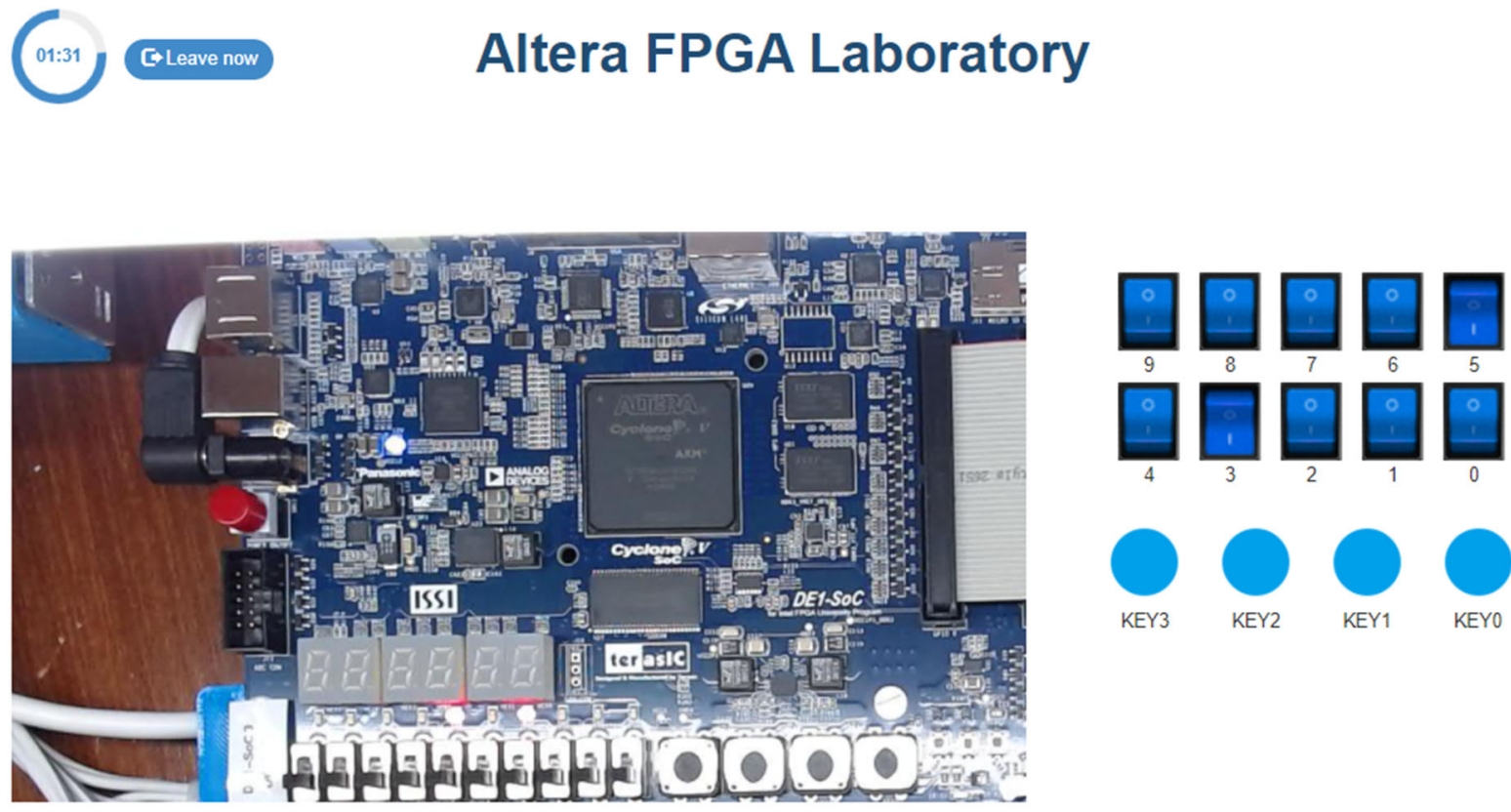

Figure 5: Labsland downloaded project running on virtualized DE1-SoC development kit showing video imagery and control switches. This FPGA development kit is hosted at the University of Washington. 
Unlike the remote console method of using the remote FPGA development kits, the user design hierarchy is unmodified, and no pin IP block is needed to memory map the switches and LEDs. The Labsland implementation captures the state of LEDs through the video camera, and stimulates the FPGA through a GPIO connection (see connector on right side of Figure 5). The FPGA development kits is connected to a Raspberry Pi board which captures mouse click from the UI and stimulates pin and switch activity on the FPGA development kit. The user must be aware that if creating their own design in Quartus, the switches/push buttons on the development kit are not utilized, and their activity is driven by the GPIO connector with pin names renamed to SW or KEY to mimic actual input behavior.

End user setup is extremely simple as no additional design tools are required to gain access to the FPGA hardware, the user simply connects to the FPGA development kit through a web interface. Labsland utilizes an instructor management console to monitor student access and usage to assist in activity and grading. Labsland is used by hundreds of labs worldwide and their scalable approach to hardware access continues to evolve into new lab topics and more universities.

Both the Intel FPGA remote console and Labsland methods of conducting remote FPGA labs are in wide use in worldwide computer engineering curriculums. The Labsland method is simpler to setup for instructors and doesn't require the remote console added level of hierarchy in the design which complicates matters for brand new users. Labsland does not currently support JTAG debug features such as the Signal Tap Logic Analyzer and In-system memory content editor, important tools that can be covered using the remote console method.

These approaches help service student communities learning electronics in a remote virtualized environment. We expect these methods to continue beyond these unprecedented times of remote learning even while we wait for the pandemic levels to subside to a level allowing a return to the in person classroom.

\section{Conclusions}

In this paper, we present several approaches that have been successfully adopted for delivering lab-based courses in online format.

The first approach presents the use of AWS cloud-based servers for Cadence tools. With minor operational modifications, this approach can be applied as a generic model for many engineering courses that have compute-intensive lab components. It's a modular cloud-based solution that can be rapidly deployed to address specific course needs without a large-scale disruption to organizational practices and policies. This AWS cloud approach entails a considerably smaller upfront expenditure. However, the overall return on investment in the AWS does not match that from a physical server over the typical life span of the infrastructure, while considering only the procurement costs of the on-premises server. In effect, for our particular use case, the traditional on-premises servers are significantly less expensive. The long-term viability of this approach is to be determined after careful consideration of all the costs involved and the advantages of the approach. 
For the second approach, we presented a cloud-based computational platform called DevCloud (by Intel). The DevCloud is an excellent platform that provides the compute resources required for heterogeneous computing courses. This free cloud service offered by Intel's Programmable Solutions Group offers Xeon processors and FPGA Platform Acceleration Cards (PAC) to universities teaching heterogeneous computing.

In addition, tried and tested approaches for remote delivery of FPGA hardware-based labs are also presented.

In effect, these approaches present options that have been successfully tried in delivering both software and hardware-based engineering labs in online format; there by satisfying a sine qua non for a frictionless transition to fully online course delivery. Even as different universities plan a phased return to in-person instruction, we feel confident in asserting that these approaches will have an enduring positive role in remote delivery of lab-based electrical engineering courses.

\section{References}

[1] "Curriculum Guidelines for Undergraduate Degree Programs in Computer Engineering," Association for Computing Machinery (ACM), IEEE Computer Society, 2016. [Online]. Available: https://www.acm.org/education/curricula-recommendations

[2] "Policy Brief: Education during COVID-19 and beyond." United Nations Organization. https://unsdg.un.org/resources/policy-brief-education-during-covid-19-and-beyond (accessed April 14, 2021).

[3] S. Khan, R. Rabbani, I. Thalassinos, and M. Atif, "Corona Virus Pandemic Paving Ways to Next Generation of Learning and Teaching: Futuristic Cloud Based Educational Model," Available at SSRN 3669832, 2020.

[4] J. Q. Michael Fullan, Max Drummy, Mag Gardner, "Education Reimagined: The Future of Learning," no. http://aka.ms/hybridlearningpaper. [Online]. Available: http://aka.ms/hybridlearningpaper

[5] "Pandemic sparks game-changing approach to hardware training." https://www.cmc.ca/pandemic-sparks-game-changing-approach-to-hardware-training/ (accessed April 14, 2021).

[6] Q. N. Naveed, M. R. N. Mohamed Qureshi, A. Shaikh, A. O. Alsayed, S. Sanober, and K. Mohiuddin, "Evaluating and Ranking Cloud-Based E-Learning Critical Success Factors (CSFs) Using Combinatorial Approach," IEEE Access, vol. 7, pp. 157145-157157, 2019, doi: 10.1109/access.2019.2949044.

[7] M. Morales Chan, R. Barchino Plata, J. A. Medina, C. Alario-Hoyos, and R. Hernandez Rizzardini, "Modeling Educational Usage of Cloud-Based Tools in Virtual Learning Environments," IEEE Access, vol. 7, pp. 13347-13354, 2019, doi: 10.1109/access.2018.2889601.

[8] A. El Mhouti, M. Erradi, and A. Nasseh, "Using cloud computing services in e-learning process: Benefits and challenges," Education and Information Technologies, vol. 23, no. 2, pp. 893-909, 2018, doi: 10.1007/s10639-017-9642-X.

[9] T. G. Peter Mell, "The NIST Definition of Cloud Computing," NIST Special Publication 800-145, 2011. 
[10]M. T. Baldassarre, D. Caivano, G. Dimauro, E. Gentile, and G. Visaggio, "Cloud Computing for Education: A Systematic Mapping Study," IEEE Transactions on Education, vol. 61, no. 3, pp. 234-244, 2018, doi: 10.1109/te.2018.2796558.

[11] L. Li, Y. Zhang, and L. Huang, "Engineering Laboratory Enhancement through Cloud Computing," ASEE Conferences, doi: 10.18260/1-2--21310. [Online]. Available: https://dx.doi.org/10.18260/1-2--21310

[12] I. Sanogo, S. Ouya, A. D. Gueye, and C. Lishou, "Proposal of cloud-based online laboratory model for practical training in the telecoms and networking fields," 2016: IEEE, doi: 10.1109/educon.2016.7474691. [Online]. Available: https://dx.doi.org/10.1109/educon.2016.7474691

[13] M. N. Kabiri and M. Wannous, "An Experimental Evaluation of a Cloud-Based Virtual Computer Laboratory Using Openstack," 2017: IEEE, doi: 10.1109/iiai-aai.2017.94. [Online]. Available: https://dx.doi.org/10.1109/iiai-aai.2017.94

[14]R. Hassan and A. A. Eman, "Cloud Computing in Computer Science and Engineering Education," San Antonio, Texas, 2012/06/10. [Online]. Available: https://peer.asee.org/21076.

[15] D. Bagchi, K. Kaushik, and B. Kapoor, "Virtual labs for electronics engineering using cloud computing," 2013: IEEE, doi: 10.1109/iedec.2013.6526757. [Online]. Available: https://dx.doi.org/10.1109/iedec.2013.6526757

[16]A. C. Caminero, S. Ros, R. Hernandez, A. Robles-Gomez, L. Tobarra, and P. J. T. Granjo, "VirTUal remoTe labORatories Management System (TUTORES): Using Cloud Computing to Acquire University Practical Skills," IEEE Transactions on Learning Technologies, vol. 9, no. 2, pp. 133-145, 2016, doi: 10.1109/tlt.2015.2470683.

[17]K.-M. Chao et al., "Cloud E-learning for Mechatronics: CLEM," Future Generation Computer Systems, vol. 48, pp. 46-59, 2015, doi: 10.1016/j.future.2014.10.033.

[18] N. Koike, "Cyber laboratory: Migration to the hybrid cloud solution for device dependent hardware experiments," 2014: IEEE, doi: 10.1109/ithet.2014.7155681. [Online]. Available: https://dx.doi.org/10.1109/ithet.2014.7155681

[19] S. Ristov, M. Gusev, and A. Donevski, "Cloud based laboratory for distance education," 2014: IEEE, doi: 10.1109/mipro.2014.6859659. [Online]. Available: https://dx.doi.org/10.1109/mipro.2014.6859659

[20] M. Wyne and J. Faraon, "Cloud Computing: Is it a way to go for Academia?," ASEE Conferences, doi: 10.18260/1-2--20168. [Online]. Available: https://dx.doi.org/10.18260/12--20168

[21] M. R. Roch, D. Demarchi, M. Klossek, and S. Tzanova, "MECA, the microelectronics cloud alliance," 2018: IEEE, doi: 10.1109/educon.2018.8363396. [Online]. Available: https://dx.doi.org/10.1109/educon.2018.8363396

[22]L. Peng, "Public and Private Clouds in Hands-on, Online Learning," Washington, District of Columbia, 2018/04/06. [Online]. Available: https://peer.asee.org/29476.

[23] T. Matthews and P. Heedley, "An Engineering Team Approach To Mentoring Graduate Students Through Projects," ASEE Conferences, doi: 10.18260/1-2--14981. [Online]. Available: https://dx.doi.org/10.18260/1-2--14981

[24]"Cadence Cloud Passport Program." Cadence. https:/www.cadence.com/en_US/home/solutions/cadence-cloud/cloud-passport.html (accessed April 14, 2021).

[25]"CMC Microsystems." https://www.cmc.ca/ (accessed April 14, 2021). 
[26]"AWS Pricing Model." https://aws.amazon.com/ec2/pricing/on-demand/ (accessed April 14, 2021).

[27]G. Deckler. "Cloud vs. On-Premises - Hard Dollar Costs." https://www.linkedin.com/pulse/cloud-vs-on-premises-hard-dollar-costs-greg-deckler (accessed May 27, 2021).

[28]"Intel® DevCloud: A Development Sandbox for Data Center to Edge Workloads." https://software.intel.com/content/www/us/en/develop/tools/devcloud.html (accessed April 14, 2021).

[29]G. Stitt. "GitHub: University Of Florida ARC." https://github.com/ARC-Lab-UF (accessed April 14, 2021).

[30]Y. Luo. "GitHub: University of Massachusetts Lowell, EECE 6540 Labs." https://github.com/acanets/eece-6540-labs (accessed April 14, 2021).

[31]H. Houman and N. Najmeh. "Hardware Accelerators using DPC++ on Intel's DevCloud." https://github.com/Najmeh-Nazari/Intel-DevCloud-FPGA-DPC- (accessed April 14, 2021).

[32]"Intel GitHub: Hands-free Labs." https://github.com/intel/FPGADevcloud/tree/master/main/HandsFree (accessed April 14, 2021).

[33]"Labs Land." https://labsland.com/en (accessed April 14, 2021).

[34] P. Orduna, L. Rodriguez-Gil, J. Garcia-Zubia, I. Angulo, U. Hernandez, and E. Azcuenaga, "LabsLand: A sharing economy platform to promote educational remote laboratories maintainability, sustainability and adoption," 2016: IEEE, doi: 10.1109/fie.2016.7757579. [Online]. Available: https://dx.doi.org/10.1109/fie.2016.7757579 